OPEN ACCESS

Edited by:

Julian Stelzer,

Case Western Reserve University,

United States

Reviewed by:

Laurin Michelle Hanft,

University of Missouri, United States

John Jeshurun Michael,

Cornell University, United States

Brett Colson,

University of Arizona, United States

${ }^{*}$ Correspondence:

Jitandrakumar R. Patel

patel@wisc.edu

Specialty section:

This article was submitted to

Striated Muscle Physiology,

a section of the journal

Frontiers in Physiology

Received: 22 August 2017 Accepted: 09 October 2017 Published: 25 October 2017

Citation:

Patel JR, Barton GP, Braun RK, Goss KN, Haraldsdottir K, Hopp A, Diffee G, Hacker TA, Moss RL and Eldridge MW (2017) Altered Right Ventricular Mechanical Properties Are

Afterload Dependent in a Rodent

Model of Bronchopulmonary

Dysplasia. Front. Physiol. 8:840. doi: 10.3389/fphys.2017.00840

\section{Altered Right Ventricular Mechanical Properties Are Afterload Dependent in a Rodent Model of Bronchopulmonary Dysplasia}

\author{
Jitandrakumar R. Patel ${ }^{* *}$, Gregory P. Barton ${ }^{2}$, Rudolf K. Braun ${ }^{2}$, Kara N. Goss², \\ Kristin Haraldsdottir ${ }^{2,3}$, Alexandria Hopp ${ }^{2,3}$, Gary Diffee ${ }^{3}$, Timothy A. Hacker ${ }^{4}$, \\ Richard L. Moss ${ }^{1}$ and Marlowe W. Eldridge ${ }^{2,3,4}$
}

${ }^{1}$ Department of Cell and Regenerative Biology, University of Wisconsin-Madison, Madison, WI, United States, ${ }^{2}$ Department of Pediatrics, University of Wisconsin-Madison, Madison, WI, United States, ${ }^{3}$ Department of Kinesiology, University of Wisconsin-Madison, Madison, WI, United States, ${ }^{4}$ Cardiovascular Research Center, University of Wisconsin School of Medicine and Public Health, Madison, WI, United States

Infants born premature are at increased risk for development of bronchopulmonary dysplasia (BPD), pulmonary hypertension $(\mathrm{PH})$, and ultimately right ventricular (RV) dysfunction, which together carry a high risk of neonatal mortality. However, the role alveolar simplification and abnormal pulmonary microvascular development in BPD affects RV contractile properties is unknown. We used a rat model of BPD to examine the effect of hyperoxia-induced $\mathrm{PH}$ on $\mathrm{RV}$ contractile properties. We measured in vivo RV pressure as well as passive force, maximum $\mathrm{Ca}^{2+}$ activated force, calcium sensitivity of force $\left(\mathrm{pCa}_{50}\right)$ and rate of force redevelopment $\left(k_{\mathrm{tr}}\right)$ in $\mathrm{R} \bigvee$ skinned trabeculae isolated from hearts of 21 -and 35 -day old rats pre-exposed to $21 \%$ oxygen (normoxia) or $85 \%$ oxygen (hyperoxia) for 14 days after birth. Systolic and diastolic RV pressure were significantly higher at day 21 in hyperoxia exposed rats compared to normoxia control rats, but normalized by 35 days of age. Passive force, maximum $\mathrm{Ca}^{2+}$ activated force, and calcium sensitivity of force were elevated and cross-bridge cycling kinetics depressed in 21-day old hyperoxic trabeculae, whereas no differences between normoxic and hyperoxic trabeculae were seen at 35 days. Myofibrillar protein analysis revealed that 21-day old hyperoxic trabeculae had increased levels of beta-myosin heavy chain $(\beta-\mathrm{MHC})$, atrial myosin light chain 1 (aMLC1; often referred to as essential light chain), and slow skeletal troponin I (ssTnl) compared to age matched normoxic trabeculae. On the other hand, 35-day old normoxic and hyperoxic trabeculae expressed similar level of $\alpha$ - and $\beta-\mathrm{MHC}$, ventricular MLC1 and predominantly CTnl. These results suggest that neonatal exposure to hyperoxia increases RV afterload and affect both the steady state and dynamic contractile properties of the RV, likely as a result of hyperoxia-induced expression of $\beta-\mathrm{MHC}$, delayed transition of slow skeletal Tnl to cardiac Tnl, and 
expression of atrial MLC1. These hyperoxia-induced changes in contractile properties are reversible and accompany the resolution of $\mathrm{PH}$ with further developmental age, underscoring the importance of reducing RV afterload to allow for normalization of RV function in both animal models and humans with BPD.

Keywords: hyperoxia, right ventricle, pulmonary hypertension, myocardial contractility, troponin isoforms; myosin heavy chain isoforms

\section{INTRODUCTION}

Infants born prematurely are at increased risk for a number of comorbidities, including the development of chronic lung disease of prematurity, or bronchopulmonary dysplasia (BPD). After preterm birth, these infants generally require resuscitation, and are often supplemented with life-sustaining oxygen therapy for prolonged periods. Exposure to a relative hyperoxic environment at a time when infants should still be in utero in a hypoxic environment has been associated with perturbed development and has long-term consequences (Jobe and Bancalari, 2001). BPD is characterized by fewer and enlarged alveoli, increased lung collagen, blunted proliferation of arterioles, increased vascular tone, decreased vascular surface area, and thickening of arterial walls (Thibeault et al., 2003; Kaarteenaho-Wiik et al., 2004; Berkelhamer et al., 2013). The development of overt pulmonary hypertension $(\mathrm{PH})$ results in increased afterload to the right ventricle (RV), leading to RV hypertrophy (RVH) and ultimately failure, with high infant morbidity and mortality (Bhat et al., 2012). Rodent models of BPD, characterized by postnatal hyperoxia exposure, recapitulate many of the findings of human disease, including arrested alveolar and vascular development, $\mathrm{PH}$ and RV dysfunction (Goss et al., 2015, 2017; Dumas de la Roque et al., 2017; Liang et al., 2017).

Recently, a study determined the effects of neonatal hyperoxia exposure on RV function in mice, demonstrating that 14-day old mice develops RVH and elevated phosphodiesterase 5 (PDE5) expression and activity, and these hyperoxia-induced changes were reversible by day 56 (Heilman et al., 2015). Indeed, previous report (Joshi et al., 2014) suggests that in later stages of childhood RV function and pulmonary arterial pressure is not different in children born preterm with existing chronic lung disease compared to term-born children who do not have chronic lung disease. However, there is limited, if any, information regarding the effects of neonatal exposure to hyperoxia on contractile properties and myofibrillar protein expression in the rodent RV. Thus, our aim was to use similar age groups of rats pre-exposed to 14 days of postnatal hyperoxia as Heilman et al. to investigate the effects of hyperoxia on in vivo RV pressure and cellular contractile properties and myofibrillar protein expression in RV. However, we chose to use 21 and 35 day old rats to investigate the effects of hyperoxia on in vivo RV pressure and cellular contractile properties and myofibrillar protein expression in RV based on our preliminary experiments, in which we were unable to isolate useable trabeculae for mechanical measurements from 14 days old rats and found mechanical properties of 35-day hyperoxic rats to be similar to aged matched normoxic rats.
We hypothesized that postnatal hyperoxia exposure in rats would result in altered RV function which will correspond with $\mathrm{PH}$.

\section{METHODS AND MATERIALS}

\section{Animals}

Timed pregnant Sprague-Dawley dams (Envigo, Indianapolis, IN) were allowed to deliver naturally at term in house. Irrespective of sex, the newborn pups were divided into two groups within $12 \mathrm{~h}$ of birth: (1) room air (normoxic), and (2) 14day hyperoxia (hyperoxic). Both groups were housed in standard cages within a $30^{\prime \prime} \times 20^{\prime \prime} \times 20^{\prime \prime}$ polypropylene chamber with a clear acrylic door. Oxygen concentration within the hyperoxia chamber was maintained at a fraction of inspired oxygen of 0.85 \pm 0.03 using a continuous oxygen sensor, while the normoxia chamber was maintained at 0.21 . Dams were rotated between room air and hyperoxia every $24 \mathrm{~h}$ to prevent oxygen-induced maternal toxicity. After 14 days, hyperoxic pups were returned to room air. Pups were weaned at 24 days. The UW School of Medicine and Public Health Animal Care and Use Committee approved all procedures involving animal care and handling.

\section{Invasive RV Pressure}

RV pressure measurements were performed at the University of Wisconsin Cardiovascular Physiology Core, as previously described (Hacker et al., 2006; Tabima et al., 2010). Briefly, 21 and 35 -day-old rats were anesthetized with urethane $(1.2 \mathrm{~g} / \mathrm{kg}$ via intraperitoneal injection), orally intubated, and mechanically ventilated (Harvard Apparatus). The chest cavity was entered through the sternum and the chest wall and lungs were gently retracted to expose the RV. A $1.9 \mathrm{~F}$ variable segment length admittance pressure catheter (Scisense, London, Ontario, Canada) was introduced into the RV using a 24-gauge needle. The magnitude and phase of the electrical E3 admittance and RV pressure were continuously recorded and analyzed using commercial software (Notocord Systems, Croissy Sur Seine, France).

\section{Preparation of Skinned Trabeculae}

Right ventricular trabeculae were isolated as described previously (Olsson et al., 2004; Patel et al., 2012). Briefly, the hearts were dissected from 21- and 35-day old normoxic and hyperoxic rats anesthetized with inhaled isoflurane. The hearts were then pinned down to the base of a dissecting dish filled with modified Ringer's solution (in mmol/L: $\mathrm{NaCl}, 120$; $\mathrm{NaHCO}_{3}, 19$; $\mathrm{Na}_{2} \mathrm{HPO}_{4}, 1.2 ; \mathrm{MgSO}_{4}, 1.2 ; \mathrm{KCl}, 5 ; \mathrm{CaCl}_{2}, 1 ;$ glucose, $10 ; \mathrm{pH}$ $7.4 ; \sim 22^{\circ} \mathrm{C}$ ) pre-equilibrated with $95 \% \mathrm{O}_{2} / 5 \% \mathrm{CO}_{2}$. After the 
right ventricles were cut open, the hearts were exposed to fresh Ringer's solution containing $20 \mathrm{mM}$ 2,3-butanedione monoxime (BDM) for $30 \mathrm{~min}$ ( $2 \mathrm{x}$ solution change). BDM was used to protect the trabeculae from cell contracture and muscle damage during dissection (Mulieri et al., 1989). Right ventricular trabeculae were then dissected free, tied to wooden applicator sticks to hold muscle length fixed, and transferred to relaxing solution (in mmol/L: $100 \mathrm{KCl}, 20$ imidazole, $7 \mathrm{MgCl}_{2}, 2 \mathrm{EGTA}$, and 4 ATP; pH $7.0 ; 4^{\circ} \mathrm{C}$ ) containing $1 \%$ Triton X-100. Following overnight skinning, the trabeculae were washed in fresh relaxing solution $(\sim 1 \mathrm{~h})$ and then stored at $-20^{\circ} \mathrm{C}$ in relaxing solution containing glycerol $(50: 50 \mathrm{v} / \mathrm{v})$. The skinned trabeculae were used in experiments within 1 week.

\section{Experimental Solutions, Apparatus, and Protocols}

Solutions composition used for mechanical measurements were calculated using the computer program of Fabiato (1988) and the stability constants (corrected to $\mathrm{pH} 7.0$ and $22^{\circ} \mathrm{C}$ ) listed by Godt and Lindley (1982). Both pCa 9.0 and pre-activating solution contained (in mmol/L) $100 \mathrm{BES}, 15$ creatine phosphate, 4.66 ATP and 5 DTT. In addition, pCa 9.0 solution contained (in $\mathrm{mmol} / \mathrm{L}$ ) 7 EGTA, $0.02 \mathrm{CaCl}_{2}, 5.49 \mathrm{MgCl}_{2}$, and 63.14 potassium propionate, whereas pre-activating solution contained (in $\mathrm{mmol} / \mathrm{L}$ ) $0.07 \mathrm{EGTA}, 5.29 \mathrm{MgCl}_{2}$, and 84.01 potassium propionate. pCa 4.5 solution contained (in mmol/L) $100 \mathrm{BES}, 15$ creatine phosphate, 4.72 ATP, 7 EGTA, $7.01 \mathrm{CaCl}_{2}, 5.29 \mathrm{MgCl}_{2}$, $5 \mathrm{DTT}$, and 49.61 potassium propionate. $\mathrm{pH}$ of all solutions was adjusted to 7.0 with $\mathrm{KOH}$. A range of $\mathrm{Ca}^{2+}$ activating solutions (pCa 6.2 to 5.4 ) were prepared by mixing solutions of $\mathrm{pCa} 9.0$ and pCa 4.5 .

On the day of an experiment, skinned trabeculae were incubated in relaxing solution for $30 \mathrm{~min}$ before cutting them free from the sticks and trimming their ends. The trimmed trabeculae were then transferred to a stainless steel experimental chamber containing pCa 9.0 solution (Moss et al., 1983). The ends of each trabecula were tied to the arms glued to a motor (model 312B, Aurora Scientific) and a force transducer (model 403; Aurora Scientific), as previously described (Moss et al., 1983). The chamber assembly was then placed on the stage of an inverted microscope (Olympus) fitted with a $40 \times$ objective and a CCTV camera (model WV-BL600, Panasonic). The light from a halogen lamp was used to illuminate the skinned preparations. Bitmap images of the preparations were acquired using an AGP $4 \mathrm{X} / 2 \mathrm{X}$ graphics card and associated software (ATI Technologies) and were used to assess mean sarcomere length (SL) during the course of each experiment. Changes in force and motor position were sampled (16-bit resolution, DAP5216a, Microstar Laboratories) at $2.0 \mathrm{kHz}$ using SLControl software developed in this laboratory (http://www.slcontrol.com). Data were saved to computer files for later analysis.

Passive force, active force-pCa, and $k_{\mathrm{tr}}-\mathrm{pCa} /$ force relationships were established at a mean SL of $\sim 2.2 \mu \mathrm{m}$ as described previously (Olsson et al., 2004; Patel et al., 2012). Briefly, the skinned trabeculae were stretched to a mean SL $\sim 2.2 \mu \mathrm{m}$ and after measuring length and width, the preparations were transferred first to pre-activating solution, then to $\mathrm{Ca}^{2+}$ activating solution, and finally back to $\mathrm{pCa} 9.0$ solution. Once in $\mathrm{Ca}^{2+}$ activated solution, steady-state force and the apparent rate constant of force redevelopment $\left(k_{\mathrm{tr}}\right)$ was measured simultaneously using the modified multi-step protocol developed by Brenner and Eisenberg (1986) as described in detail previously (Patel et al., 2001) and illustrated in Figure 1. Briefly, after force reached a steady level in activating solution (pCa 6.2-4.5), the length of the preparation was rapidly reduced by $\sim 20 \%$, held for $\sim 20 \mathrm{~ms}$, and then re-stretched back to its original length. As a result, there was an initial transient increase, followed by a decrease in force (seen as a spike in the force trace) and subsequent slower recovery of force to near the initial steady-state level. $k_{\text {tr }}$ reported in the present study is the rate constant of force redevelopment after the spike. The drop in force recorded in solution of $\mathrm{pCa} 9.0$ was considered to be passive force and was therefore subtracted from the drop in total force at each pCa to yield $\mathrm{Ca}^{2+}$ activated force $(\mathrm{P})$. The protocol was repeated to establish active force-pCa and $k_{\mathrm{tr}}$-pCa/relative active force relationships. After completing mechanical measurements, the trabeculae were detached from the points of attachment, placed in sodium dodecyl sulfate (SDS) sample buffer (8M Urea, $2 \mathrm{M}$ thiourea, $0.05 \mathrm{M}$ Tris pH6.8, $75 \mathrm{mM}$ DTT, 3\% SDS, and $0.01 \%$ bromophenol blue) and stored at $-80^{\circ} \mathrm{C}$ until subsequent protein analysis.

\section{SDS Page Silver Stain Protein Content Analysis}

To examine the expression profile of MHC isoforms, samples were prepared using RV free wall isolated from normoxic and hyperoxic treated rats and gels were prepared as described previously (Warren and Greaser, 2003). Briefly, RV free wall was homogenized in relaxing solution and homogenate washed with fresh relaxing solution. Next, the homogenate was incubated for $30 \mathrm{~min}$ in relaxing solution containing 1\% Triton-100. After the incubation period, the homogenate was washed three times with relaxing solution and $2.4 \mathrm{mg}$ (wet weight) of homogenate was suspended in $100 \mu \mathrm{L}$ SDS sample buffer and stored at $-80^{\circ} \mathrm{C}$ until subsequent protein analysis. A $17 \mathrm{~mL}$ solution of resolving gel $(6 \% \mathrm{~T}$ and $2 \% \mathrm{C})$ was prepared by mixing $8.27 \mathrm{~mL}$ water, $1.7 \mathrm{ml} 50 \%$ glycerol (v/v), $2.55 \mathrm{~mL} 40 \%$ acrylamide (37.5:1 crosslinked with DATD), $4.24 \mathrm{~mL} 1.5 \mathrm{M}$ Tris, $\mathrm{pH} 8.8,170 \mu \mathrm{L} 10 \%$ SDS (w/v), $50 \mu \mathrm{L} 10 \%$ ammonium persulfate (w/v), and $20 \mu \mathrm{L}$ TEMED. The resolving gel was poured into an empty BioRad Criterion cassette and water was added to the top of the resolving gel to form a flat surface. After an hour of polymerization, the gel was stored in the cold room overnight. Next day, water was drained out and stacking gel $(3 \% \mathrm{~T}$ and $1.5 \% \mathrm{C} ; 1.15 \mathrm{~mL}$ water, $1 \mathrm{ml} 50 \%$ glycerol $(\mathrm{v} / \mathrm{v}), 1.5 \mathrm{~mL} 10 \%$ acrylamide $(5.6: 1$ cross-linked with DATD), $1.3 \mathrm{~mL} 0.5 \mathrm{M}$ Tris, $\mathrm{pH} 6.8,50 \mu \mathrm{L}$ $10 \% \operatorname{SDS}(\mathrm{w} / \mathrm{v}), 30 \mu \mathrm{L} 10 \%$ ammonium persulfate $(\mathrm{w} / \mathrm{v})$, and $25 \mu \mathrm{L}$ TEMED) was poured over the resolving gel. A 12 well comb was inserted and stacking gel was allowed to polymerize for an hour. The gel cassette was then inserted into a BioRad Criterion gel box pre-filled with ice cold lower running buffer (25.09 mM Tris-base, $19.98 \mathrm{mM}$ glycine, $3.47 \mathrm{mM}$ SDS, and 


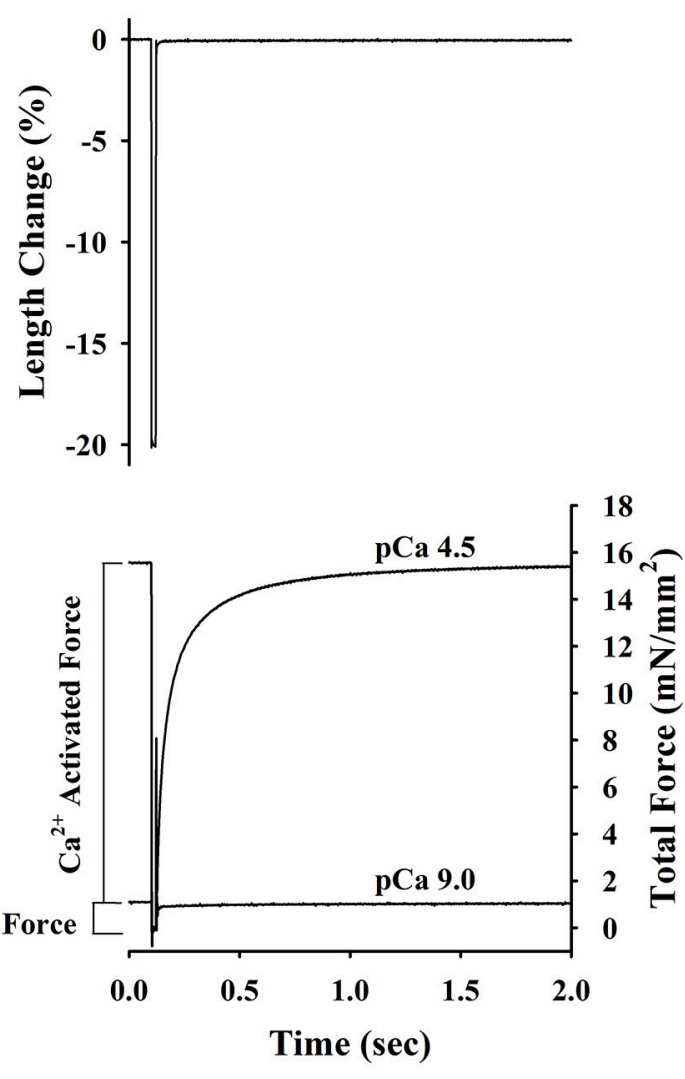

FIGURE 1 | Experimental protocol for determining of passive force, $\mathrm{Ca}^{2+}$-activatedforce and rate constant of force redevelopment $\left(k_{\mathrm{tr}}\right)$ in rat skinned right ventricular trabecula. The bottom panel shows the changes in force recorded before, during and after a step change in length (top panel) of a rat skinned right ventricular trabecula. Once active force reached a steady state in pCa 4.5 and 9.0 solutions, muscle length was rapidly slackened by $20 \%$, held at this length for $20 \mathrm{~ms}$ and finally re-stretched back to its original length. The $\mathrm{Ca}^{2+}$-activated force was determined by subtracting the resting force measured at $\mathrm{pCa} 9.0$ from the total force measured at $\mathrm{pCa} 4.5 . \mathrm{ktr}$ is the apparent rate constant of force redevelopment following re-stretch of trabeculae to its original length.

$2 \mathrm{mM}$ 2-mecaptoethanol). The comb was removed, wells were washed with water and the chamber was filled with ice cold upper running buffer (50.18 mM Tris-base, $39.96 \mathrm{mM}$ glycine, $6.94 \mathrm{mM}$ SDS, and $10 \mathrm{mM}$ 2-mecaptoethanol). The samples were defrosted and $4 \mu \mathrm{L}$ of sample was added to $36 \mu \mathrm{L}$ of sample buffer. The diluted sample was heated $\left(95^{\circ} \mathrm{C}\right)$ for $3 \mathrm{~min}$ and allowed to cool down before loading $10 \mu \mathrm{L}$ on to the gel. Electrophoresis was done at $16 \mathrm{~mA}$ constant current for $4 \mathrm{~h}$ in the cold room. At the end of electrophoresis, the gel was removed from the cassette and silver stained using method described previously (Shevchenko et al., 1996) with following modification (Stelzer et al., 2006). The gels were (a) incubated overnight in fixing solution containing $50 \%$ methanol and $10 \%$ acetic acid, (b) washed for $20 \mathrm{~min}(4 \mathrm{x}$ $\mathrm{ddH}_{2} \mathrm{O}$ changes) with $\mathrm{ddH}_{2} \mathrm{O}$, (c) incubated for $1.5 \mathrm{~min}$ in $0.01 \%$ sodium thiosulfate solution and then rinsed $4 \mathrm{x}$ with $\mathrm{ddH}_{2} \mathrm{O}$, (d) incubated for $20 \mathrm{~min}$ in $0.09 \%$ silver nitrate solution and then rinsed $4 \mathrm{x}$ with $\mathrm{ddH}_{2} \mathrm{O}$, (e) incubated in developing solution containing $0.0004 \%$ sodium thiosulfate, $2 \%$ potassium carbonate, and $0.0068 \%$ formaldehyde until protein bands were visible and then rinsed $4 \mathrm{x}$ with $\mathrm{ddH}_{2} \mathrm{O}$, (f) incubated for $20 \mathrm{~min}$ in destaining solution containing $10 \%$ methanol and $10 \%$ acetic

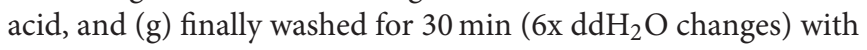
dd $\mathrm{H}_{2} \mathrm{O}$.

To examine expression profile of myofibrillar proteins, the normoxic and hyperoxic trabeculae stored in SDS sample buffer were electrophoresed using 12\% Tris- $\mathrm{HCl}$ Precast Criterion gels (BioRad, Hercules, CA). The gel cassette was inserted into BioRad Criterion gel box pre-filled with running buffer $(25.09 \mathrm{mM}$ Trisbase, $19.98 \mathrm{mM}$ glycine, $3.47 \mathrm{mM}$ SDS). The comb was removed, wells were washed with water and the chamber was filled with running buffer. The samples were defrosted, sonicated for $15 \mathrm{~min}$ and $8 \mu \mathrm{L}$ of sample was loaded on to the gel. Electrophoresis was done at constant volts $(150 \mathrm{~V})$ for $1.5 \mathrm{~h}$ at room temperature. At the end of the run, the gels were removed from the cassette and silver stained as described above. Both gels were imaged and protein bands quantified using BioRad Chemi Doc MP Imaging System (BioRad, Hercules, CA).

\section{Data Analysis and Statistics}

Cross-sectional areas of skinned trabeculae were calculated by assuming that the trabeculae were cylindrical and by equating the width, measured from video images of the mounted preparations, to diameter. Each $\mathrm{Ca}^{2+}$ activated force $(\mathrm{P})$ at $\mathrm{pCa}$ between 6.2 and 5.4 was expressed as a fraction of the maximum $\mathrm{Ca}^{2+}$ activated force $\left(\mathrm{P}_{\mathrm{o}}\right)$ developed by the same preparations at $\mathrm{pCa}$ 4.5 , i.e., $\mathrm{P} / \mathrm{P}_{\mathrm{o}}$. To determine the $\mathrm{Ca}^{2+}$ sensitivity of isometric force $\left(\mathrm{pCa}_{50}\right)$, force-pCa data were fitted with the Hill equation: $\mathrm{P} / \mathrm{P}_{\mathrm{o}}$ $\left.=\left[\mathrm{Ca}^{2+}\right]^{\mathrm{n}} /\left(k^{\mathrm{n}}+\left[\mathrm{Ca}^{2+}\right]^{\mathrm{n}}\right)\right]$, where $\mathrm{n}$ is the slope (Hill coefficient) and $k$ is the $\mathrm{Ca}^{2+}$ concentration for half-maximal activation $\left(\mathrm{pCa}_{50}\right) . k_{\mathrm{tr}}$ was determined by linear transformation of the halftime of force recovery $\left(k_{\mathrm{tr}}=-\ln 0.5 \times\left(\mathrm{t}_{1 / 2}\right)^{-1}\right)$, as described previously (Chase et al., 1994; Patel et al., 2001). All data are presented as means \pm standard error (SE). Statistical analysis of the RV pressure data was performed using two-way ANOVA (age and group main effects; GraphPad Prism 6; GraphPad Software Inc., San Diego, CA), all other analysis with unpaired $t$-tests (Sigma Plot 11.Ink; Systat Software Inc., San Jose, CA), p-values $<0.05$ were taken as indicating significant differences.

\section{RESULTS}

\section{Recovery of RV Pressure from Day 21 to Day 35 in Hyperoxia Exposed Rats}

A total of 49 rats were exposed to postnatal normoxia, and 40 rats were exposed to postnatal hyperoxia. Sixteen normoxia and hyperoxia exposed rats were used for the determination of RV pressures at 21 and 35 days of age ( $n=8$ in each group), respectively. There was a significant difference $(p<0.05)$ in body weight at day $21(51.4 \pm 1.4$ vs. $42.4 \pm 1.6 \mathrm{~g})$ but no difference in body weight at day $35(127.0 \pm 4.7$ vs. $117.1 \pm 3.2 \mathrm{~g})$ in normoxia treated vs. hyperoxia treated rats, respectively. The systolic and diastolic RV pressure (Figure 2) was significantly higher in the hyperoxic group at day 21 compared to the normoxic group at the same time point. This difference between the normoxic and 


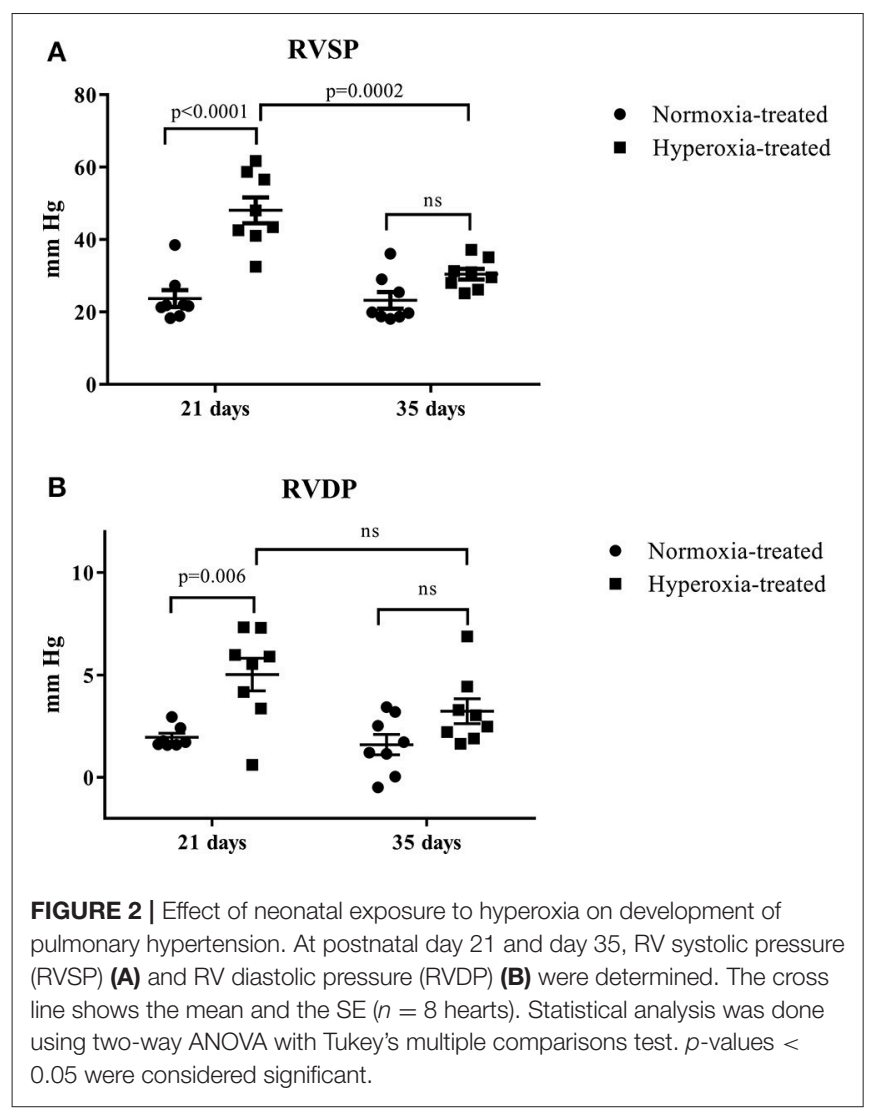

hyperoxic group resolved by day 35 with $\mathrm{RV}$ pressure in the hyperoxic group being significantly lower compared to day 21 . This suggests a recovery of the hyperoxia-induced $\mathrm{PH}$ seen at day 21 .

\section{Effects of Hyperoxia Exposure on Passive Force, Maximum $\mathrm{Ca}^{2+}$ Activated Force, Apparent Cooperativity in Activation of Force $\left(n_{H}\right)$, and $\mathrm{Ca}^{2+}$ Sensitivity of Force $\left(\mathrm{pCa}_{50}\right)$}

Steady-state mechanical properties were assessed using right ventricular trabeculae isolated from a second cohort of 21-day normoxic ( $n=18$ trabeculae/15 rats) and hyperoxic ( $n=16$ trabeculae/12 rats) and 35-day normoxic ( $n=15$ trabeculae/8 rats) and hyperoxic ( $n=9$ trabeculae/8 rats) (Table 1 ). At pCa 9.0, passive force generated by 21-day hyperoxic trabeculae was almost twice $(p<0.001)$ than age matched normoxic trabeculae, and by 35-day hyperoxic trabeculae generated similar amount of passive force as age matched normoxic trabeculae (Table 1). At pCa 4.5, maximum $\mathrm{Ca}^{2+}$ activated force generated by 21 -day hyperoxic trabeculae was $\sim 70 \%$ greater $(p=$ 0.002 ) than age matched normoxic trabeculae, and by 35 -day hyperoxic trabeculae were similar to age matched normoxic trabeculae (Table 1). At sub-maximal $\mathrm{Ca}^{2+}$ (pCa 6.2-5.4), 21day hyperoxic trabeculae also generated more force than age matched normoxic trabeculae, resulting in a left shift of hyperoxic trabeculae sigmodal force-pCa relationships compared to age matched normoxic trabeculae (Figure 3A). Fitting the force-pCa relationships with the Hill equation yielded significantly higher $\mathrm{pCa}_{50}$ values $\left(\Delta \mathrm{pCa}_{50}=0.23 ; p<0.001\right)$, implying elevated $\mathrm{Ca}^{2+}$ sensitivity of force, and lower $\mathrm{n}_{\mathrm{H}}$ values $\left(\Delta \mathrm{n}_{\mathrm{H}}=0.3\right.$; $p=0.005$ ), which implies depressed apparent cooperativity in activation of force, for hyperoxic compared to normoxic trabeculae. On the other hand, sub-maximal forces generated by 35 -day hyperoxic trabeculae were similar to those measured in age matched normoxic trabeculae and as a result there was no discernable difference between the sigmodal force-pCa relationships established in hyperoxic and age matched normoxic trabeculae (Figure 3B). Fitting the force-pCa relationships with the Hill equation yielded similar $\mathrm{pCa}_{50}$ values and $\mathrm{n}_{\mathrm{H}}$ values for hyperoxic and age matched normoxic trabeculae. These results suggest that the neonatal exposure to hyperoxia has profound early effects on steady-state force production and that these effects wane as the $\mathrm{PH}$ resolves.

\section{Effects of Hyperoxia on Rate of Force Redevelopment $\left(\mathbf{k}_{\mathrm{tr}}\right)$}

Irrespective of age, both normoxic and hyperoxic trabeculae exhibited $\left[\mathrm{Ca}^{2+}\right]_{\text {free }}$ (and force)-dependent changes in the rate of force redevelopment $\left(k_{\mathrm{tr}}\right)$, confirming earlier results from rat (Wolff et al., 1995; Palmer and Kentish, 1998; Patel et al., 2012) myocardium. That is, increasing the $\left[\mathrm{Ca}^{2+}\right]_{\text {free }}$ from $\mathrm{pCa}$ 6.2 to $\mathrm{pCa} 4.5$ elevated the values of $k_{\mathrm{tr}}$ from $2.62 \pm 0.14$ to $10.48 \pm 1.08 \mathrm{~s}^{-1}$ and $1.93 \pm 0.21$ to $8.28 \pm 1.02 \mathrm{~s}^{-1}$ in 21 day normoxic and hyperoxic trabeculae and from $2.61 \pm 0.12$ to $11.74 \pm 0.58 \mathrm{~s}^{-1}$ and $2.79 \pm 0.17$ to $10.43 \pm 0.78 \mathrm{~s}^{-1}$ in 35-day normoxic and hyperoxic trabeculae. To illustrate this, records of force redevelopment at various levels of $\left[\mathrm{Ca}^{2+}\right]_{\text {free }}$ are shown in Figure 4 for 21-day normoxic (Figure 4A) and hyperoxic (Figure 4B) and 35-day normoxic (Figure 4C) and hyperoxic (Figure 4D) trabecula, where steady state forces at each pCa were normalized to 1.0 to provide better visualization of variations in kinetics of force redevelopment. Figure 5 shows the curvilinear $k_{\mathrm{tr}}$-relative force relationships observed in 21-day (Figure 5A) and 35-day (Figure 5B) normoxic and hyperoxic trabeculae. At pCa 4.5, both 21 and 35-day hyperoxic trabeculae redeveloped maximum $\mathrm{Ca}^{2+}$ activated force at similar rates as age matched normoxic trabeculae. At sub-maximal free $\left[\mathrm{Ca}^{2+}\right]$ (pCa 6.2-5.4), 21-day hyperoxic trabeculae redeveloped submaximal forces at significantly slower rates than age matched normoxic trabeculae and as a result the curvilinear $k_{\mathrm{tr}}$-relative force relationships established in hyperoxic trabeculae were to the right of those established in normoxic trabeculae (Figure 5A). On the other hand, 35-day hyperoxic trabeculae redeveloped sub-maximal forces at rates similar to age matched normoxic trabeculae and as a result there was no discernable difference between curvilinear $k_{\mathrm{tr}}$-relative force relationships established in hyperoxic and age matched normoxic trabeculae (Figure 5B). These results indicate that the neonatal exposure to hyperoxia has profound effects on cross-bridge cycling kinetics and that the effects are reversible in rat myocardium. These reversible effects are coincident with the normalization of RV pressure at 35 days. 
TABLE 1 | Summary of mechanical properties of skinned right ventricular trabeculae isolated from 21-and 35-day old Normic and Hyperoxic rat myocardium.

\begin{tabular}{|c|c|c|c|c|c|}
\hline Treatment & $\begin{array}{l}\text { Passive force } \\
\left(\mathrm{mN} / \mathrm{mm}^{2}\right)\end{array}$ & $\begin{array}{l}\text { Maximum } \mathrm{Ca}^{2+} \text { activated force } \\
\qquad\left(\mathrm{mN} / \mathrm{mm}^{2}\right)\end{array}$ & Hill coefficient $\left(n_{H}\right)$ & $\begin{array}{l}\mathrm{Ca}^{2+} \text { sensitivity of } \\
\text { force }\left(\mathrm{pCa}_{50}\right)\end{array}$ & $\begin{array}{l}\text { Maximum rate of force } \\
\text { redevelopment }\left(k_{\mathrm{tr}}, \mathbf{s}^{-1}\right)\end{array}$ \\
\hline \multicolumn{6}{|l|}{ 21-DAY OLD } \\
\hline Hyperoxic (16/12) & $2.69 \pm 0.29^{\star}$ & $22.05 \pm 1.30^{*}$ & $2.26 \pm 0.08^{*}$ & $5.87 \pm 0.02^{\star}$ & $8.28 \pm 1.02$ \\
\hline \multicolumn{6}{|l|}{ 35-DAY OLD } \\
\hline
\end{tabular}

All values are expressed as means $\pm S E$, with number of skinned right ventricular trabeculae/rat hearts given in parentheses. At SL of $2.2 \mu m$, passive force was measured at pCa 9.0. Maximum force and the rate constant of force redevelopment $\left(k_{t r}\right)$ were measured at $p C a$ 4.5. $p C a_{50}$ and $n_{H}$ values were derived by fitting the force-pCa relationships to a Hill equation. "Significantly different $(p<0.05)$ from values recorded in age matched normoxic skinned trabeculae.
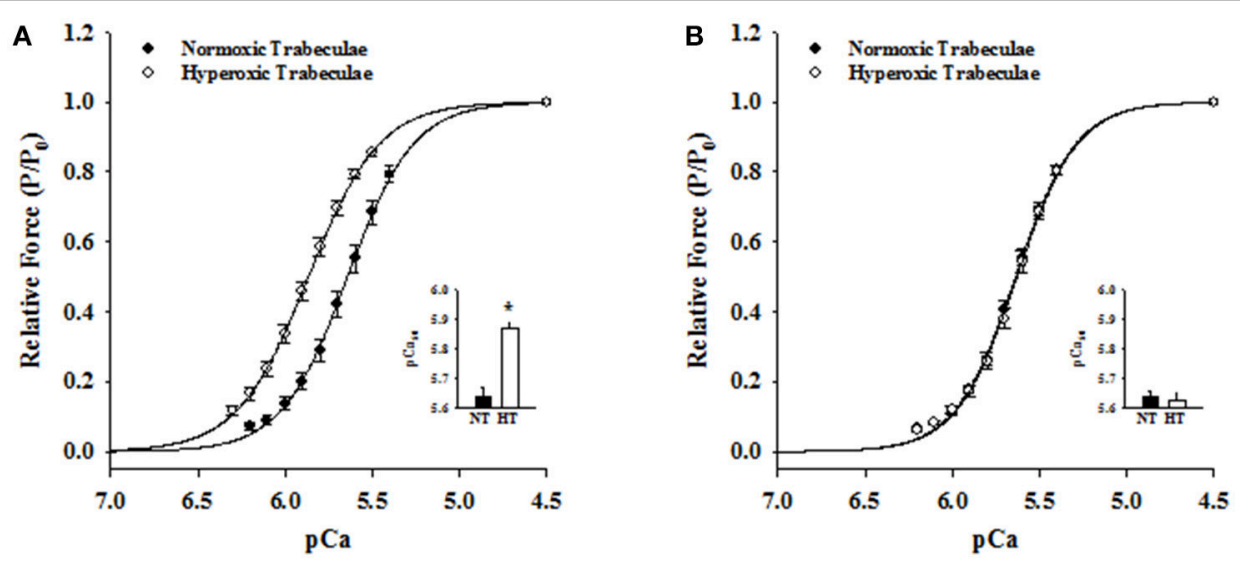

FIGURE 3 | Effects of neonatal exposure to hyperoxia on apparent cooperativity in the activation of force $\left(\mathrm{n}_{H}\right)$ and the $\mathrm{Ca}^{2+}$ sensitivity of force (pCa50) in 21 - and 35-day skinned right ventricular trabeculae. Force-pCa relationships were measured in 21-Day normoxic (closed circles; $n=18$ trabeculae from 15 hearts) and hyperoxic (open circles; 16 trabeculae from 12 hearts) trabeculae (A) and 35-Day normoxic (closed circles; $n=15$ trabeculae from 8 hearts) and hyperoxic (open circles; 9 trabeculae from 8 hearts) trabeculae (B). Submaximal $\mathrm{Ca}^{2+}$ activated forces at $\mathrm{pCa}$ 6.2-5.4 were expressed as a fraction of maximum Ca ${ }^{2+}$ activated force at $\mathrm{pCa}$ 4.5. The solid lines were generated by fitting the mean data with the Hill equation described in Methods and Materials. The respective fitted values of $\mathrm{n}_{\mathrm{H}}$, apparent cooperativity of activation of force, and $\mathrm{pCa}_{50}$, an index for $\mathrm{Ca}^{2+}$ sensitivity of force, for 21-day normoxic trabeculae were 2.29 and pCa 5.64 and for hyperoxic trabeculae were 2.13 and pCa 5.87 (A). The respective fitted values of $\mathrm{n}_{\mathrm{H}}$ and $\mathrm{pCa} 50$ for 35 -day normoxic trabeculae were 2.51 and pCa 5.64 and for hyperoxic trabeculae were 2.51 and pCa 5.63 (B). Inset shows the $\mathrm{pCa}_{50}$ values determined in normoxic (black bars) and hyperoxic (white bars) trabeculae. Data points are the means \pm SE. *Significantly different $(p<0.05)$ from values determined in normoxic trabeculae.

\section{Effects of Hyperoxia on Myofibrillar Protein Expression}

Figure 6 shows a typical SDS-PAGE analysis of MHC isoforms (Figures 6A,B) and myofibrillar protein expression (Figures 6C,D) in 21- and 35-day normoxic and hyperoxic myocardium. Figures 6A,B shows that 21-day hyperoxic RV expressed $14 \%$ less $\alpha$-MHC and more $\beta$-MHC than aged matched normoxic RV. Whereas, 35-day hyperoxic RV expressed similar levels of both $\alpha$-MHC and $\beta$-MHC as aged matched normoxic RV. It is also apparent from Figure 6C that both 21- and 35-day hyperoxic trabeculae expressed similar isoforms of key myofibrillar proteins as normoxic trabeculae (cardiac myosin binding protein C (cMyBP-C), actin, troponin $\mathrm{T}(\mathrm{TnT})$, tropomyosin $(\mathrm{Tm})$, ventricular myosin light chain 1 , and 2 (vMLC1 and vMLC2), respectively. While both 21 and 35-day normoxic trabeculae were found to express $100 \%$ cTnI, an observation consistent with a previous study which reported complete conversion of ssTnI to cTnI by 15 days after birth in rat (Warren et al., 2004), 21-day hyperoxic trabeculae expressed both cTnI $(33 \pm 2 \%)$ and ssTnI $(67 \pm 2 \%)$ (Figure 6D). With the exception of one hyperoxic trabeculae expressing both cTnI (31\%) and ssTnI (69\%), 35-day normoxic and hyperoxic rat trabeculae expressed predominately cTnI. In addition, 21-day, but not 35-day, hyperoxic trabeculae expressed aMLC1 (15 $\pm 3 \%)$ and vMLC1 $(85 \pm 3 \%$ ) (Figure 6D), an observation consistent with $\mathrm{PH}$-induced expression of aMLC1in neonatal porcine right ventricle (Morano et al., 1998). These results suggest that neonatal exposure to hyperoxia elevates expression of $\beta$-MHC, disrupts transition of ssTnI to cTnI, stimulates expression of aMLC1, and indicates that most of these effects are reversible in rat myocardium.

\section{DISCUSSION}

The goal of the present study was to use skinned right ventricular trabeculae isolated from hearts of 21 and 35-day old rats 
A

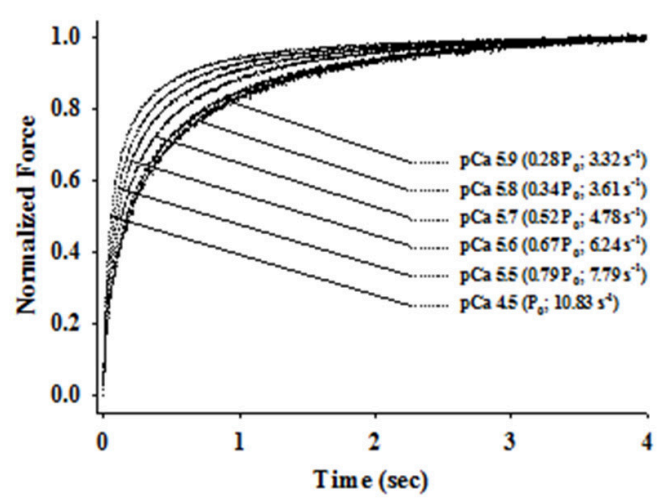

C

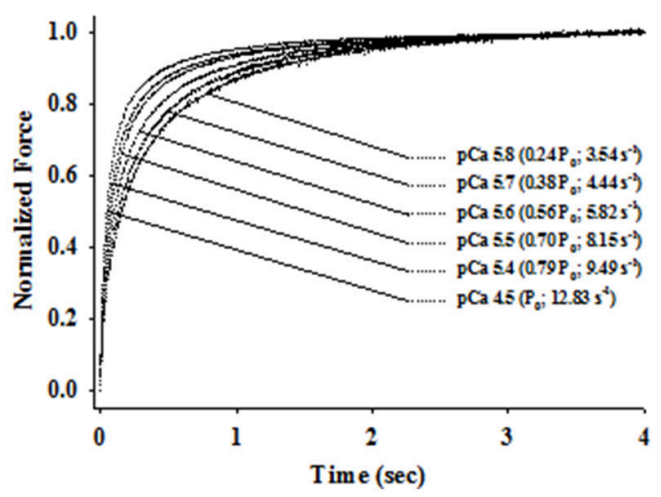

B 21-Day Hyperoxic T rabecula

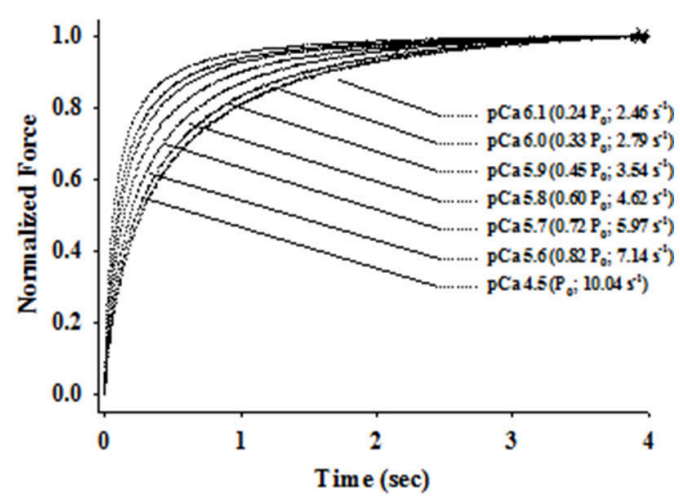

D

35-Day Hyperoxic T rabecula

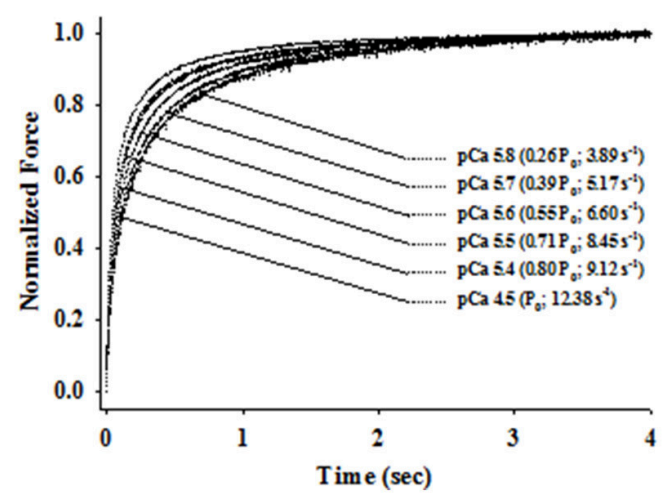

FIGURE 4 | $\mathrm{Ca}^{2+}$ - and force-dependent changes in apparent rate constant of force redevelopment $\left(k_{\mathrm{tr}}\right)$ in 21 - and 35 -Day normoxic and hyperoxic skinned right ventricular trabecula. The rate of force redevelopment $\left(k_{\mathrm{tr}}\right)$ was measured as described in Methods and Materials at sub-maximal (pCa 6.1-5.4) and maximum (pCa 4.5) $\left[\mathrm{Ca}^{2+}\right]_{\text {free }}$ in skinned right ventricular trabecula isolated from 21-Day normoxic (A) and hyperoxic (B) and 35-Day normoxic (C) and hyperoxic (D) rat ventricle. The force transients were expressed relative to the peak steady state force attained after the step change in muscle length. Both, relative force and $k_{\mathrm{tr}}$ values for a given $\left[\mathrm{Ca}^{2+}\right]_{\text {free }}$ is shown in parentheses.
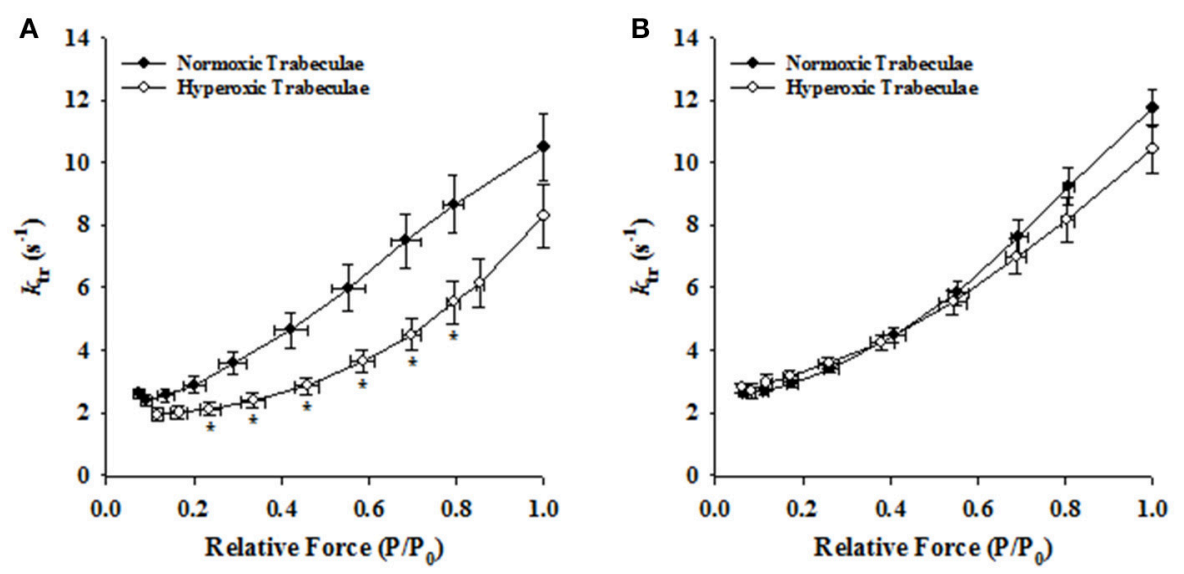

FIGURE 5 | Effects of neonatal exposure to hyperoxia on the rate of force redevelopment $\left(k_{\mathrm{tr}}\right)$ as a function of relative force $\left(\mathrm{P} / \mathrm{P}_{0}\right)$ relationships in 21 - and 35 -day

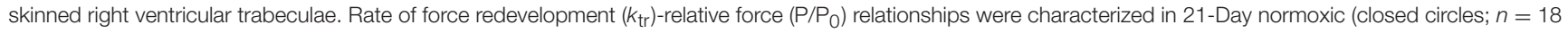
trabeculae from 15 hearts) and hyperoxic (open circles; 16 trabeculae from 12 hearts) trabeculae (A) and 35-Day normoxic (closed circles; $n=15$ trabeculae from 8 hearts) and hyperoxic (open circles; 9 trabeculae from 8 hearts) trabeculae (B). ${ }^{*}$ Significantly different $(p<0.05)$ from values determined in normoxic trabeculae. 

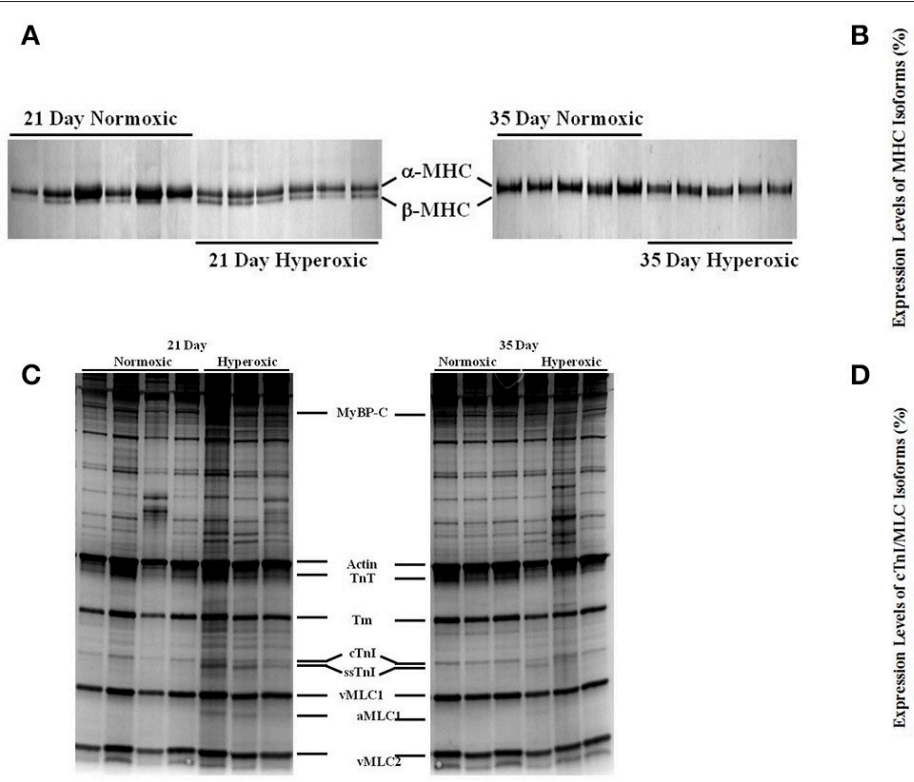

D
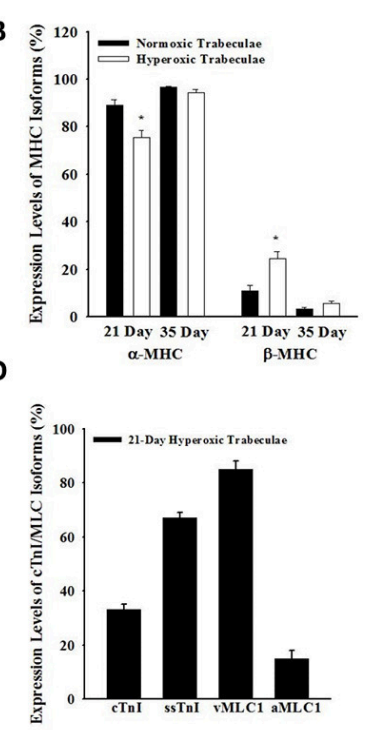

FIGURE 6 | Effects of neonatal exposure to hyperoxia on myofibrillar protein compositions in 21- and 35-day skinned right ventricular trabeculae. A typical expression profile of myosin heavy chain isoforms ( $\mathrm{MHC}$ ) in 6 normoxic and hyperoxic 21-day right ventricles and 5 normoxic and hyperoxic 35-day right ventricles (A) and other myofilament proteins in four normoxic and hyperoxic 21-day skinned right ventricular trabeculae and 3 normoxic and hyperoxic 35-day skinned right ventricular trabeculae (C) examined by SDS-PAGE as described in Methods and Materials. Myofibrillar proteins are identified in order of increasing mobility. (B) 21-day hyperoxic myocardium was expressing significantly lower $\alpha-\mathrm{MHC}$ and higher $\beta-\mathrm{MHC}$ than normoxic myocardium, whereas, 35-day hyperoxic and normoxic myocardium were expressing similar levels of both $\alpha$ - and $\beta$-MHC. (D) 21-day hyperoxic trabeculae were expressing both cTnl and ssTnl, and vMLC1 and a-MLC1 isoforms. Beside above differences, there were no other major detectable differences in the expression profiles of myofibrillar proteins between normoxic and hyperoxic trabeculae. CMyBP-C, cardiac myosin binding protein-C; TnT, troponin T; Tm, tropomyosin; CTnl, cardiac troponin I; ssTnl, slow skeletal Troponin I; vMLC1, ventricular myosin light chain 1; aMLC1, atrial myosin light chain 1; vMLC2, ventricular myosin light chain 2. *Significantly different $(p<0.05)$ from values determined in normoxic right ventricular wall.

to examine the impact of neonatal hyperoxia exposure on contractile properties and protein expression within the RV in a model of BPD. We demonstrated for the first time that neonatal exposure to hyperoxia has profound effects on passive force (increase), maximum $\mathrm{Ca}^{2+}$ activated force (increase), $\mathrm{Ca}^{2+}$ sensitivity of force (increase), apparent cooperativity in activation of force (decrease), cross-bridge cycling kinetics (slower), expression of $\beta$-MHC (higher), aMLC1, and the developmental transition of ssTnI to cTnI (delayed). Furthermore, these effects of neonatal exposure to hyperoxia on steady-state force production, cross-bridge cycling kinetics and protein expression normalize as RV afterload and $\mathrm{PH}$ improve by 35 days of age. Together these changes result in a hypercontractile RV as a neonatal adaptive response to hyperoxia-induced $\mathrm{PH}$.

Chronic lung disease of prematurity, or BPD, is frequently complicated by $\mathrm{PH}$, which results in a significant increase in neonatal morbidity and mortality (Baker et al., 2014). We chose to use rats at the ages of 21 and 35-days postnatal life which corresponds with weaning ( $\sim 6$ months of age) and prepubertal human stages of life, respectively (Sengupta, 2013). A previous study reported that preterm infants with $\mathrm{PH}$ and $\mathrm{BPD}$ demonstrated a mortality of $38 \%$ during a median follow up of 10.9 months (Khemani et al., 2007). However, the majority of individuals born preterm with chronic lung disease have a normalization of $\mathrm{RV}$ function and $\mathrm{PA}$ pressure in later childhood despite persistently abnormal lung function (Joshi et al., 2014). Our data demonstrates a similar finding to humans in which altered RV function and RV pressure in early stages of development normalizes with further developmental age. Furthermore, these findings of mechanical disruption of the RV is coincident with $\mathrm{PH}$ at 21 days postnatal rat life corresponds with a time in life where humans born preterm diagnosed with $\mathrm{BPD}$ and PH are most susceptible to higher mortality rates.

\section{Impact of Hyperoxia on Steady-State Contractile Properties of Right Ventricle}

The 21-day hyperoxic trabeculae generated twice as much force as normoxic trabeculae (Table 1), an effect similar to the $\mathrm{PH}$-induced increase in maximum $\mathrm{Ca}^{2+}$ activated force observed in the adult human (Rain et al., 2013), rat (Kogler et al., 2003), and mice (unpublished observation). While these results are consistent with the idea that higher density of thick and thin filaments allows 21-day hyperoxic trabeculae to generate more force, the finding of similar amount of maximum $\mathrm{Ca}^{2+}$ activated forces in 35-day hyperoxic and normoxic trabeculae is inconsistent with this mechanism. Previous studies reported that myocardium expressing ssTnI generates a maximum $\mathrm{Ca}^{2+}$ activated force similar to myocardium expressing cTnI (Fentzke et al., 1999; Arteaga et al., 2000; Konhilas et al., 2003; Ford and Chandra, 2012) whereas expression of aMLC1 results in a higher maximum $\mathrm{Ca}^{2+}$ activated force than expression of vMLC1 (Morano et al., 1998). 
Interestingly, our findings of increased maximum $\mathrm{Ca}^{2+}$ activated force and aMLC1 in 21-day old hyperoxia exposed rats was associated with $\mathrm{PH}$, which is similar to a report in a porcine model of PH (Morano et al., 1998). Taken together, the earlier observation of increased maximum $\mathrm{Ca}^{2+}$ activated force in 21-day hyperoxic trabeculae and the similar amount of maximum $\mathrm{Ca}^{2+}$ activated force generated by 35 -day hyperoxic trabeculae compared to normoxic trabeculae suggests that expression of aMLC1 is likely to play a prominent role in increasing maximum $\mathrm{Ca}^{2+}$ activated force in 21-day hyperoxic trabeculae.

At sub-maximal $\mathrm{Ca}^{2+}, 21$-day hyperoxic trabeculae generated more force than age matched normoxic trabeculae and as a result, the force-pCa relationships established in hyperoxic trabeculae were left-shifted by $\sim 0.23 \mathrm{pCa}$ units compared to those in normoxic trabeculae (Figure $\mathbf{3 A}$ ), which corresponds to similar changes found in monocrotaline-induced $\mathrm{PH}$ (Kogler et al., 2003). In cardiac muscle, expression of either ssTnI (Fentzke et al., 1999; Arteaga et al., 2000; Konhilas et al., 2003; Ford and Chandra, 2012) or aMLC1 (Morano et al., 1997; Diffee and Nagle, 2003; Diffee, 2004) are known to shift force-pCa relationships to the left. Since 21-day hyperoxic trabeculae expressed both ssTnI and aMLC1, it is difficult to say with certainty that the increased $\mathrm{Ca}^{2+}$ sensitivity of force in 21-day hyperoxic trabeculae was due to the presence of ssTnI or aMLC1, or both. However, the finding of similar $\mathrm{Ca}^{2+}$ sensitivities of force in 35-day hyperoxic trabeculae and age-matched normoxic trabeculae (Figure 3B), in which there is no aMLC1 present and predominant expression of cTnI (Figure 6C), suggest that the hyperoxia-induced changes in expression of MLC1/TnI isoforms at 21 days is reversible and may be responsible for the increase in $\mathrm{Ca}^{2+}$ sensitivity in hyperoxic myocardium at this age. Taken together, our findings of increased maximum $\mathrm{Ca}^{2+}$ activated force, $\mathrm{Ca}^{2+}$ sensitivity of force and changes in expression of MLC1/TnI isoforms at 21 days of age in our hyperoxia exposed rats are likely an adaptive response to $\mathrm{PH}$. This is highlighted by the findings at 35 days of age where there were no differences in RV pressure, RV myofibrillar isoform expression and contractile properties.

\section{Impact of Hyperoxia on Dynamic Contractile Properties of Right Ventricle}

In normoxic trabeculae, the rate of force redevelopment $\left(k_{\mathrm{tr}}\right)$ varied with the level of activating $\left[\mathrm{Ca}^{2+}\right]_{\text {free }}$ (or force), increasing as $\left[\mathrm{Ca}^{2+}\right]_{\text {free }}$ (or force) was elevated from sub-maximal to maximal levels (Figures 4, 5). These $\mathrm{Ca}^{2+}$ - and force-dependent changes in $k_{\mathrm{tr}}$ in normoxic trabeculae are consistent with previous results from rat (Wolff et al., 1995; Palmer and Kentish, 1998; Olsson et al., 2004; Patel et al., 2012), mice (Edes et al., 2007; Colson et al., 2012; Ford and Chandra, 2012), porcine (Edes et al., 2007) and human (Edes et al., 2007) myocardium. Both, 35-day normoxic and hyperoxic trabeculae also exhibited similar $\mathrm{Ca}^{2+}$ and force-dependent changes in $k_{\mathrm{tr}}$, suggesting no remaining significant effects of hyperoxia on these relationships. However, 21-day hyperoxic trabeculae redeveloped sub-maximal forces at a slower rate than age-matched normoxic trabeculae (Figure 4).
Thus, when $k_{\mathrm{tr}}$ values were plotted against force normalized to maximum force, $k_{\mathrm{tr}}$-force relationships in hyperoxic trabeculae were right-shifted compared to those in normoxic trabeculae (Figure 5A), i.e., at equivalent forces, $k_{\mathrm{tr}}$ values were lower in hyperoxic trabeculae. In adult rat myocardium, a decrease in expression of $\alpha-\mathrm{MHC}$, and concomitant increase in expression of $\beta$-MHC, is known to slow the rate of force redevelopment (Fitzsimons et al., 1999; Rundell et al., 2005; Locher et al., 2011) and rate of relaxation (Fitzsimons et al., 1998). Thus, the possibility of the depressed cross-bridge cycling kinetics in 21day hyperoxic trabeculae may be exclusively or in part due to a decrease in expression of $\alpha-\mathrm{MHC}$ and concomitant increase in expression of $\beta$-MHC (Figure 6B). Previous studies have reported that expression of ssTnI has no significant effects on the rate of force redevelopment in skinned preparations (Ford and Chandra, 2012), whereas expression of aMLC1 increases contraction and relaxation time in whole heart (Morano et al., 1996, 1998; Fewell et al., 1998; Abdelaziz et al., 2004). Since there is expression of aMLC1 and ssTnI in 21-day hyperoxic myocardium but not in normoxic myocardium, both aMLCland ssTnI appears to be responsible for slower contraction kinetics in hyperoxic myocardium at this age.

Alternatively, the ability of hyperoxic trabeculae to generate more force than normoxic trabeculae can be explained in the context of a two-state kinetic model of cross-bridge interaction proposed by Huxley (1957) and modified by Brady (1991). In this model, multiple states of the cross-bridge kinetic scheme are reduced to just two states, i.e., the transition from nonforce-generating to force-generating states is described by $f_{a p p}$, whereas $g_{a p p}$ describes the transition from the force-generating state back to the non-force-generating state. Steady isometric force (P) is then equal to $N \times F \times\left[f_{a p p} /\left(f_{a p p}+g_{a p p}\right)\right]$, where $N$ is the number of cycling cross-bridges, $F$ is the average force per cross-bridge and $k_{\mathrm{tr}}=f_{\mathrm{app}}+g_{\mathrm{app}}$. Thus, the hyperoxiainduced increase in $\mathrm{Ca}^{2+}$ sensitivity of force in the present study may be due to an increase in $N$, or $F$, or the proportion of cross-bridges in the force generating state as a result of an increase in $f_{a p p}$, a decrease $g_{a p p}$, or both. An increase in either the probability of myosin cross-bridge binding to actin (in case of aMLC1; Schaub et al., 1998) or the binding affinity of TnC for $\mathrm{Ca}^{2+}$ (in case of ssTnI) would increase $\mathrm{N}$ and facilitate cooperative binding of myosin cross-bridges to actin. The latter would be manifested as a decrease in $\mathrm{n}_{\mathrm{H}}$ (an index of apparent cooperativity in activation of force; Table 1) of the force-pCa relationship, and a decreased cross-bridge detachment rate $\left(g_{a p p}\right)$, which would be manifested as decrease in $k_{\mathrm{tr}}$. Interestingly, $g_{a p p}$ derived from natural logarithm of $k_{\mathrm{tr}}$-relative force relationship (data not shown) was lower in 21-day hyperoxic $\left(1.44 \mathrm{~s}^{-1}\right)$ than normoxic $\left(2.17 \mathrm{~s}^{-1}\right)$ trabeculae. Thus, it appears that the combined effect of $\beta$-MHC, ssTnI (increased binding affinity of TnC for $\mathrm{Ca}^{2+}$ ) and aMLC1 (increased probability of myosin cross-bridge binding to actin) are important for the reduced kinetics in the RV at 21 days of age. Although, no previous studies have explored cross-bridge cycling kinetics in a model of BPD, we can infer that reduced cross-bridge cycling kinetics in 21 day hyperoxia exposed rats is likely due to the adaptive response to pulmonary pressure overload and the subsequent 
myofibrillar isoform expression changes at 21 days of age in this model.

\section{CONCLUSION}

In summary, we found hyperoxia-induced changes in expression of MHC, TnI, and MLC1 isoforms are reversible upon normalization of RV pressure and responsible for altering both steady-state force production and crossbridge cycling kinetics in rat myocardium. Increasing developmental age in this rodent model of BPD is associated with a reversal of $\mathrm{PH}$-induced $\mathrm{RV}$ dysfunction, which has also been observed in humans with BPD associated with preterm birth. This work underscores the importance of reducing RV afterload to allow for recovery of RV function in both animal models and humans with BPD.

\section{REFERENCES}

Abdelaziz, A. I., Segaric, J., Bartsch, H., Petzhold, D., Schlegel, W. P., Kott, M., et al. (2004). Functional characterization of the human atrial essential myosin light chain (hALC-1) in a transgenic rat model. J. Mol. Med. 82, 265-274. doi: 10.1007/s00109-004-0525-4

Arteaga, G. M., Palmiter, K. A., Leiden, J. M., and Solaro, R. J. (2000). Attenuation of length dependence of calcium activation in myofilaments of transgenic mouse hearts expressing slow skeletal troponin I. J. Physiol. 526(Pt 3), 541-549. doi: 10.1111/j.1469-7793.2000.t01-1-00541.x

Baker, C. D., Abman, S. H., and Mourani, P. M. (2014). Pulmonary hypertension in preterm infants with bronchopulmonary dysplasia. Pediatr. Allergy Immunol. Pulmonol. 27, 8-16. doi: 10.1089/ped.2013.0323

Berkelhamer, S. K., Mestan, K. K., and Steinhorn, R. H. (2013). Pulmonary hypertension in bronchopulmonary dysplasia. Sem. Perinatol. 37, 124-131. doi: 10.1053/j.semperi.2013.01.009

Bhat, R., Salas, A. A., Foster, C., Carlo, W. A., and Ambalavanan, N. (2012). Prospective analysis of pulmonary hypertension in extremely low birth weight infants. Pediatrics 129, e682-e689. doi: 10.1542/peds.2011-1827

Brady, A. J. (1991). Mechanical properties of isolated cardiac myocytes. Physiol. Rev. 71, 413-428.

Brenner, B., and Eisenberg, E. (1986). Rate of force generation in muscle: correlation with actomyosin ATPase activity in solution. Proc. Natl. Acad. Sci. U.S.A. 83, 3542-3546. doi: 10.1073/pnas.83.10.3542

Chase, P. B., Martyn, D. A., and Hannon, J. D. (1994). Isometric force redevelopment of skinned muscle fibers from rabbit activated with and without $\mathrm{Ca}^{2+}$. Biophys. J. 67, 1994-2001. doi: 10.1016/S0006-3495(94)80682-4

Colson, B. A., Patel, J. R., Chen, P. P., Bekyarova, T., Abdalla, M. I., Moss, R. L., et al. (2012). Myosin binding protein-C phosphorylation is the principal mediator of protein kinase A effects on thick filament structure in myocardium. J. Mol. Cell. Cardiol. 53, 609-616. doi: 10.1016/j.yjmcc.2012.07.012

Diffee, G. M. (2004). Adaptation of cardiac myocyte contractile properties to exercise training. Exerc. Sport Sci. Rev. 32, 112-119. doi: 10.1097/00003677-200407000-00007

Diffee, G. M., and Nagle, D. F. (2003). Regional differences in effects of exercise training on contractile and biochemical properties of rat cardiac myocytes. J. Appl. Physiol. 95, 35-42. doi: 10.1152/japplphysiol.00951.2002

Dumas de la Roque, E., Smeralda, G., Quignard, J. F., Freund-Michel, V., Courtois, A., Dubois, M., et al. (2017). Altered vasoreactivity in neonatal rats with pulmonary hypertension associated with bronchopulmonary dysplasia: implication of both eNOS phosphorylation and calcium signaling. PLOS ONE 12:e0173044. doi: 10.1371/journal.pone.0173044

Edes, I. F., Czuriga, D., Csanyi, G., Chlopicki, S., Recchia, F. A., Papp, Z., et al. (2007). Rate of tension redevelopment is not modulated by sarcomere length

\section{AUTHOR CONTRIBUTIONS}

JP: Designed and conducted experiments, analyzed data and wrote the manuscript; GB: analyzed data, wrote and edited the manuscript; RB: Conducted experiments and edited the manuscript; KG: Contributed to design, wrote and edited the manuscript; $\mathrm{KH}$ : Conducted experiments; $\mathrm{AH}$ : Conducted experiments; GD: edited the manuscript; TH: Conducted experiments, analyzed data and edited the manuscript; RM: Oversaw experiments, wrote and edited the manuscript; ME: Contributed to design, oversaw experiments, wrote and edited the manuscript.

\section{FUNDING}

This work was supported by funding from the NIH-NHLBI R01 HL115061-03S1 (Eldridge).

in permeabilized human, murine, and porcine cardiomyocytes. Am. J. Physiol. 293, R20-R29. doi: 10.1152/ajpregu.00537.2006

Fabiato, A. (1988). Computer programs for calculating total from specified free or free from specified total ionic concentrations in aqueous solutions containing multiple metals and ligands. Methods Enzymol. 157, 378-417. doi: 10.1016/0076-6879(88)57093-3

Fentzke, R. C., Buck, S. H., Patel, J. R., Lin, H., Solaro, R. J., Leinden, J. M., et al. (1999). Impared cardiomyocyte relaxation and diastolic function in transgenic mice expressing slow skeletal troponin I in the heart. J. Physiol. 517, 143-157. doi: 10.1111/j.1469-7793.1999.0143z.x

Fewell, J. G., Hewett, T. E., Sanbe, A., Klevitsky, R., Hayes, E., Robbins, J., et al. (1998). Functional significance of cardiac myosin essential light chain isoform switching in transgenic mice. J. Clin. Invest. 101, 2630-2639. doi: 10.1172/JCI2825

Fitzsimons, D. P., Patel, J. R., and Moss, R. L. (1999). Aging-dependent depression in the kinetics of force development in rat skinned myocardium. Am. J. Physiol. 276(5 Pt 2), H1511-H1519.

Fitzsimons, D. P., Patel, J. R., and Moss, R. L. (1998). Role of myosin heavy chain composition in kinetics of force development and relaxation in rat myocardium. J. Physiol. 513(Pt 1), 171-183. doi: 10.1111/j.1469-7793.1998.171by.x

Ford, S. J., and Chandra, M. (2012). The effects of slow skeletal troponin I expression in the murine myocardium are influenced by developmentrelated shifts in myosin heavy chain isoform. J. Physiol. 590, 6047-6063. doi: 10.1113/jphysiol.2012.240085

Godt, R. E., and Lindley, B. D. (1982). Influence of temperature upon contractile activation and isometric force production in mechanically skinned muscle fibers of the frog. J. Gen. Physiol. 80, 279-297. doi: 10.1085/jgp.80.2.279

Goss, K. N., Cucci, A. R., Fisher, A. J., Albrecht, M., Frump, A., Lahm, V., et al. (2015). Neonatal hyperoxic lung injury favorably alters adult right ventricular remodeling response to chronic hypoxia exposure. Am. J. Physiol. Lung Cell. Mol. Physiol. 308, L797-L806. doi: 10.1152/ajplung.00276.2014

Goss, K. N., Kumari, S., Tetri, L. H., Barton, G., Braun, R. K., Eldridge, M. W., et al. (2017). Postnatal hyperoxia exposure durably impairs right ventricular function and mitochondrial biogenesis. Am. J. Respir. Cell Mol. Biol. 56, 609-619. doi: 10.1165/rcmb.2016-0256OC

Hacker, T. A., McKiernan, S. H., Douglas, P. S., Wanagat, J., and Aiken, J. M. (2006). Age-related changes in cardiac structure and function in Fischer 344 x Brown Norway hybrid rats. Am. J. Physiol. Heart Circ. Physiol. 290, H304-H311. doi: 10.1152/ajpheart.00290.2005

Heilman, R. P., Lagoski, M. B., Lee, K. J., Taylor, J. M., Kim, G. A., Farrow, K. N., et al. (2015). Right ventricular cyclic nucleotide signaling is decreased in hyperoxia-induced pulmonary hypertension in neonatal mice. Am. J. Physiol. 308, H1575-H1582. doi: 10.1152/ajpheart.00569.2014 
Huxley, A. F. (1957). Muscle structure and theories of contraction. Prog. Biophys. Biophys. Chem. 7, 255-318.

Jobe, A. H., and Bancalari, E. (2001). Bronchopulmonary dysplasia. Am. J. Respir. Crit. Care Med. 163, 1723-1729. doi: 10.1164/ajrccm.163.7.2011060

Joshi, S., Wilson, D. G., Kotecha, S., Pickerd, N., Fraser, A. G., and Kotecha, S. (2014). Cardiovascular function in children who had chronic lung disease of prematurity. Arch. Dis. Child. Fetal Neonatal Ed. 99, F373-F379. doi: 10.1136/archdischild-2013-305185

Kaarteenaho-Wiik, R., Paakko, P., Risteli, J., and Soini, Y. (2004). Type I and III collagen protein precursors and mRNA in the developing human lung. J. Pathol. 203, 567-574. doi: 10.1002/path.1547

Khemani, E., McElhinney, D. B., Rhein, L., Andrade, O., Lacro, R. V., Mullen, M. P., et al. (2007). Pulmonary artery hypertension in formerly premature infants with bronchopulmonary dysplasia: clinical features and outcomes in the surfactant era. Pediatrics 120, 1260-1269. doi: 10.1542/peds.2007-0971

Kogler, H., Hartmann, O., Leineweber, K., Nguyen van, P., Schott, P., Brodde, O. E., et al. (2003). Mechanical load-dependent regulation of gene expression in monocrotaline-induced right ventricular hypertrophy in the rat. Circ. Res. 93, 230-237. doi: 10.1161/01.RES.0000085042.89656.C7

Konhilas, J. P., Irving, T. C., Wolska, B. M., Jweied, E. E., Martin, A. F., Solaro, R. J., et al. (2003). Troponin I in the murine myocardium: influence on lengthdependent activation and interfilament spacing. J. Physiol. 547(Pt 3), 951-961. doi: 10.1113/jphysiol.2002.038117

Liang, Z. J., Wu, Q. P., Chen, B. T., Lin, Z. L., Lin, J., and Chen, S. Q. (2017). Postnatal hyperoxia or DEHP exposure leads to growth restriction and delayed lung development in newborn rats. Pediatr. Neonatol. doi: 10.1016/j.pedneo.2016.11.002. [Epub ahead of print].

Locher, M. R., Razumova, M. V., Stelzer, J. E., Norman, H. S., and Moss, R. L. (2011). Effects of low-level a-myosin heavy chain expression on contractile kinetics in porcine myocardium. Am. J. Physiol. 300, H869-H878. doi: 10.1152/ajpheart.00452.2010

Morano, I., Hadicke, K., Haase, H., Bohm, M., Erdmann, E., and Schaub, M. C. (1997). Changes in essential myosin light chain isoform expression provide a molecular basis for isometric force regulation in the failing human heart. J. Mol. Cell. Cardiol. 29, 1177-1187. doi: 10.1006/jmcc.1996.0353

Morano, M., Boels, P., Haworth, S. G., Haase, H., and Morano, I. (1998). Expression and function of atrial myosin light chain 1 in the porcine right ventricle of normal and pulmonary hypertensive animals. Adv. Exp. Med. Biol. 453, 481-488. doi: 10.1007/978-1-4684-6039-1_53

Morano, M., Zacharzowski, U., Maier, M., Lange, P. E., Alexi-Meskishvili, V., Morano, I., et al. (1996). Regulation of human heart contractility by essential myosin light chain isoforms. J. Clin. Invest. 98, 467-473. doi: $10.1172 /$ JCI1 18813

Moss, R. L., Swinford, A. E., and Greaser, M. L. (1983). Alterations of the Ca2+ sensitivity of tension development by single skeletal muscle fibers at stretched lengths. Biophys. J. 43, 115-119. doi: 10.1016/S0006-3495(83)84329-X

Mulieri, L. A., Hasenfuss, G., and Ittleman, F. (1989). Blanchard EM, Alpert NR. Protection of human left ventricular myocardium from cutting injury with 2,3-butanedione monoxime. Circ. Res. 65, 1441-1449.

Olsson, M. C., Patel, J. R., Fitzsimons, D. P., Walker, J. W., and Moss, R. L. (2004). Basal myosin light chain phosphorylation is a determinant of $\mathrm{Ca}^{2+}$ sensitivity of force and activation dependence of the kinetics of myocardial force development. Am. J. Physiol. 287, H2712-H2718. doi: 10.1152/ajpheart.01067.2003

Palmer, S., and Kentish, J. C. (1998). Roles of $\mathrm{Ca}^{2+}$ and crossbridge kinetics in determining the maximum rates of $\mathrm{Ca}^{2+}$ activation and relaxation in rat and guinea pig skinned trabeculae. Circ. Res. 83, 179-186. doi: 10.1161/01.RES.83.2.179

Patel, J. R., Fitzsimons, D. P., Buck, S. H., Muthuchamy, M., Wieczorek, D. F., and Moss, R. L. (2001). PKA accelerates rate of force development in murine skinned myocardium expressing $\alpha$ - or $\beta$-tropomyosin. Am. J. Physiol. 280, H2732-H2739.

Patel, J. R., Pleitner, J. M., Moss, R. L., and Greaser, M. L. (2012). Magnitude of length-dependent changes in contractile properties varies with titin isoform in rat ventricles. Am. J. Physiol. 302, H697-H708. doi: 10.1152/ajpheart.00800.2011

Rain, S., Handoko, M. L., Trip, P., Gan, C. T., Westerhof, N., Stienen, G. J., et al. (2013). Right ventricular diastolic impairment in patients with pulmonary arterial hypertension. Circulation 128, 1-10. doi: 10.1161/CIRCULATIONAHA.113.001873

Rundell, V. L. M., Manaves, V., Martin, A. F., and de Tombe, P. P. (2005). Impact of $\beta$-myosin heavy chain isoform expression on cross-bridge cycling kinetics. Am. J. Physiol. 288, H896-H903. doi: 10.1152/ajpheart.00407.2004

Schaub, M. C., Hefti, M. A., Zuellig, R. A., and Morano, I. (1998). Modulation of contractility in human cardiac hypertrophy by myosin essential light chain isoforms. Cardiovasc. Res. 37, 381-404. doi: 10.1016/S0008-6363(97)00258-7

Sengupta, P. (2013). The laboratory rat: relating its age with human's. Int. J. Prev. Med. 4, 624-630.

Shevchenko, A., Wilm, M., Vorm, O., and Mann, M. (1996). Mass spectrometric sequencing of proteins from silver-stained polyacrylamide gels. Anal. Chem. 68, 850-858. doi: 10.1021/ac950914h

Stelzer, J. E., Patel, J. R., and Moss, R. L. (2006). Acceleration of stretch activation in murine myocardium due to phosphorylation of myosin regulatory light chain. J. Gen. Physiol. 128, 261-272. doi: 10.1085/jgp.200609547

Tabima, D. M., Hacker, T. A., and Chesler, N. C. (2010). Measuring right ventricular function in the normal and hypertensive mouse hearts using admittance-derived pressure-volume loops. Am. J. Physiol. Heart Circ. Physiol. 299, H2069-H2075. doi: 10.1152/ajpheart.00805.2010

Thibeault, D. W., Truog, W. E., and Ekekezie I. I. (2003). Acinar arterial changes with chronic lung disease of prematurity in the surfactant era. Pediatr. Pulmonol. 36, 482-489. doi: 10.1002/ppul.10349

Warren, C. M., and Greaser, M. L. (2003). Method for cardiac myosin heavy chain deperation by sodium dodecyl sulfate gel elecrophoresis. Anal. Biochem. 320, 149-151. doi: 10.1016/S0003-2697(03)00350-6

Warren, C. M., Krzesinski, P. R., Campbell, K. S., Moss, R. L., and Greaser, M. L. (2004). Titin isoform changes in rat myocardium during development. Mech. Dev. 121, 1301-1312. doi: 10.1016/j.mod.2004.07.003

Wolff, M. R., McDonald, K. S., and Moss, R. L. (1995). Rate of tension development in cardiac muscle varies with level of activator calcium. Circ. Res.. 76, 154-160. doi: 10.1161/01.RES.76.1.154

Conflict of Interest Statement: The authors declare that the research was conducted in the absence of any commercial or financial relationships that could be construed as a potential conflict of interest.

Copyright $\odot 2017$ Patel, Barton, Braun, Goss, Haraldsdottir, Hopp, Diffee, Hacker, Moss and Eldridge. This is an open-access article distributed under the terms of the Creative Commons Attribution License (CC BY). The use, distribution or reproduction in other forums is permitted, provided the original author(s) or licensor are credited and that the original publication in this journal is cited, in accordance with accepted academic practice. No use, distribution or reproduction is permitted which does not comply with these terms. 